\title{
Synthesis and Catalytic Application of Zeolite-Based Hollow Materials
}

\author{
Xiangping $\mathrm{Li}^{1,2}$, Hongpeng Zhang ${ }^{1,2}$, Yuan Fang ${ }^{1}$, Sen Lin ${ }^{1}$, Liang Song ${ }^{1}$, \\ Guangci $\mathrm{Li}^{1}$, Hongchuan Xin ${ }^{1, *}$, and Xuebing $\mathrm{Li}^{1, *}$ \\ ${ }^{1}$ Key Laboratory of Biofuels, Qingdao Institute of Bioenergy and Bioprocess Technology, \\ Chinese Academy of Sciences, Qingdao 266101, China \\ ${ }^{2}$ University of Chinese Academy of Sciences, Beijing 100049, China
}

\section{ABSTRACT}

Owing to the diffusion limitations of reactants and products in micropores, large zeolite crystals are not suitable for catalytic reactions involving macromolecules. Hierarchically porous zeolites with mesopores or macropores exhibit remarkably improved catalytic performance. Moreover, zeolite-based hollow structures are attracting a great deal of interest for their diverse applications in the areas of biochemistry, photonics, and catalysis. This review presents the fabrication and catalytic applications of the various zeolite-based hollow materials. The synthesis methods are classified to three categories, and the formation mechanisms were briefly summarized in this paper.

KEYWORDS: Zeolite-Based Hollow Materials, Synthesis, Catalytic Application.

\section{CONTENTS}

2. Fabrication and Catalytic Application of Zeolite-Based Hollow Materials ... . . . . . . . . . . . . . . . . . . . . . . . . . . 259

2.1. Fabrication .................... 259

2.2. Catalytic Applications ... . . . . . . . . . . 263

3. Conclusion ... . . . . . . . . . . . . . . . . . . 264

Acknowledgment . . . . . . . . . . . . . . . . . . . . . . . 264

References and Notes . . . . . . . . . . . . . . . . . . . . . 264

\section{INTRODUCTION}

Due to its unique properties such as large micro-porosity, molecular sieving behavior and high hydrothermal stability, zeolite has been widely used as catalyst in oil refining ${ }^{1}$ and chemicals production via aromatization, ${ }^{2}$ alkylation, ${ }^{3}$ and catalytic cracking. ${ }^{4}$ However, the intrinsic microporous channels in micron-sized zeolite impose diffusion limitations. Large hydrocarbon molecules retained within micropores deactivate zeolite catalysts due to pore blockage or coking. In the past decades, nano-sized zeolite (nano-zeolite) has gradually offered its great potentials not only in catalysis and adsorption but also in a wide variety of new applications. The small size of nanozeolite crystals can reduce the mass transport limitation of guest molecules in the micropore, and thereby leads to a remarkable catalytic performance and high coking-tolerant

\footnotetext{
*Authors to whom correspondence should be addressed.

Emails: xinhc@qibebt.ac.cn, lixb@qibebt.ac.cn

Received: 18 February 2014

Accepted: 7 March 2014
}

ability. Besides, the rich external surface characteristics of nano-zeolite also provide it with new opportunities in the fields of immobilization, separation, and identification of biomolecules. However, the difficult manipulation of nano-zeolite particles is a pending obstacle in most of their applications, whereas the conventional moulding and aggregation procedures in industry inevitably cause the lessening of accessible active surface. ${ }^{5}$

Recently, several methods have been developed to prepare hierarchical zeolitic materials with various macroscopic morphologies including membranes, ${ }^{6-8}$ sphere, ${ }^{9}$ monoliths, ${ }^{10-14}$ and zeolitic composites, ${ }^{15,16}$ etc., which might provide more benefits in the practical application of zeolites. ${ }^{17-19}$ Many artificial materials, such as cellulose acetate membrane, ${ }^{8}$ polystyrene (PS) spheres, ${ }^{10}$ carbon fibers, ${ }^{20,21}$ and mesoporous silica spheres ${ }^{13,22}$ have been used as templates to control the product structure and morphology.

Hollow inorganic microcapsules with a tiny shell and a relatively large core space, which have the ability of storage of various species, have potential applications in many fields such as controlled release capsules, host materials, coatings, confined-space catalysts, bioreactors and other functional materials. ${ }^{23}$ Zeolites are the ideal building blocks to construct hollow capsules because they may introduce the uniform micropores on the shell, which would be beneficial for shape-selective catalysis and components delivery. Extensive attention has been paid to the preparation of zeolite hollow spheres, and a number of methodologies have been exploited, e.g., the carbon 

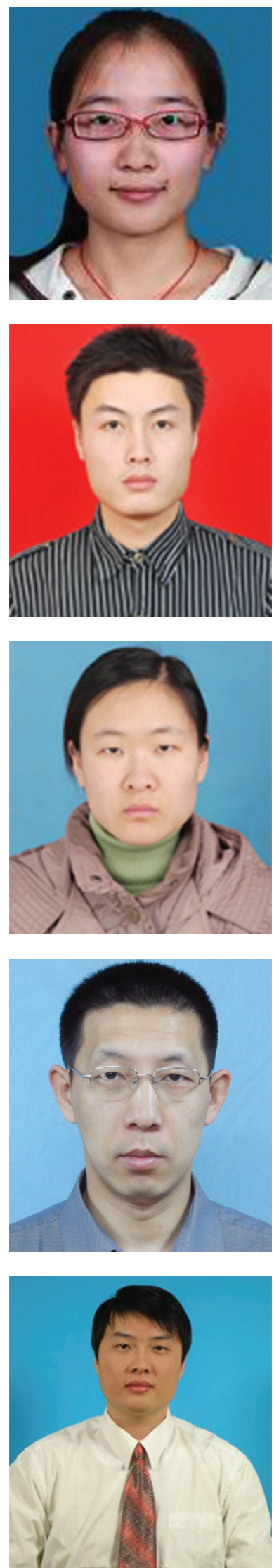

Xiangping Li was born in Shandong, China, in 1985. She received her B.S. and M.S. degrees in college of chemical engineering from Qingdao University of Science and Technology in 2008 and 2011, respectively. She is now pursuing her doctor degree at Qingdao Institute of Bioenergy and Bioprocess Technology, Chinese Academy of Sciences. Her main research interests are the application of catalysts and catalytic processes for bioethanol dehydration to ethylene.

Hongpeng Zhang was born in Shandong, China, in 1988. He received his B.S. degree in college of chemical and environmental engineering from Shandong University of Science and Technology in 2011. He is now pursuing his master degree at Qingdao Institute of Bioenergy and Bioprocess Technology, Chinese Academy of Sciences since 2011. His main research interests are the catalyst and catalytic process for ethylene oligomerization.

Yuan Fang was born in Jiangsu, China, in 1988. She received her B.S. degree in School of Petrochemical Engineering from Changzhou University in 2012. She is now pursuing her master degree at Changzhou University since 2012. Her main research interests are the catalyst and catalytic process for bioethanol dehydration to ethylene.

Sen Lin is an Associate Professor of Qingdao Institute of Bioenergy and Bioprocess Technology, Chinese Academy of Science (CAS). He received his Ph.D. in Jilin University, (2005) with major in Inorganic Synthesis and Preparative Chemistry. Followed he conducted postdoc research in Shanghai Research Institute of Petrochemical Technology, Shanghai (2005-2006) and EcoTopia Science Institute, Nagoya University (2006-2007). After that he was financially supported by Fundação para a Ciência e a Tecnologia (FCT), worked in Évora Chemistry Center, Évora University, Portugal (2007-2010). His research interests are inorganic material and heterogeneous catalysis.

Liang Song was born in Hebei, China, in 1982. He received his B.S. degree in College of Materials Science and Engineering from University of Jinan in 2004, his M.E. degree in College of Materials Science and Engineering from Guangxi University in 2007 and his Ph.D. degree in College of Chemistry and Chemical Engineering from Ocean University of China in 2011. At the end of 2009, he was as a joint training doctoral student studied in the Oak Ridge National Laboratory in USA for a year. His mainly research interests are the biomass transformation to energy, such as catalytic conversion of biological lactic acid and lignin, the synthesis of biodiesel, etc. 

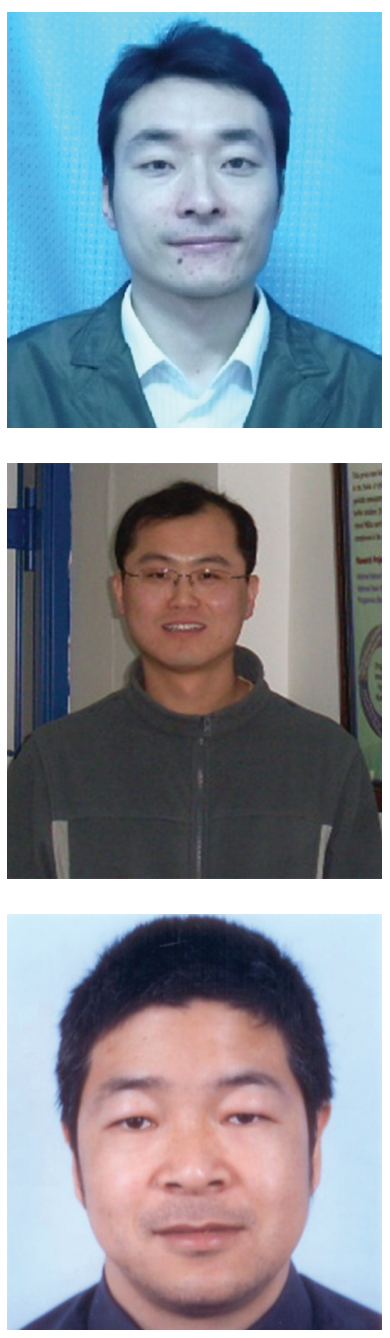

Xuebing Li was born in Tianjin, China, in 1972. He received his B.S. and M.S. degrees in Department of Chemistry from Tsinghua University in 1995 and 1998, respectively, and his Ph.D degree in Technical Chemistry at TU München in 2004. He worked as postdoctoral researcher at UC Berkeley (2005-2007) and Senior Scientist at TU München (2007-2010). He was appointed as a full professor in 2010 at Qingdao Institute of Bioenergy and Bioprocess Technology, Chinese Academy of Sciences. His main research interest is heterogeneous catalytic conversion of substantially available raw materials to modern energy products. He is also extensively exploring the reaction mechanisms, micro-kinetics and catalytically active surface structures.

black hard template self-assembly combined hydrothermal crystallization process, layer-by-layer (LBL) self-assembly technique, self-assembly of nanocrystals with the aid of sonication in ammoniac ethanol, etc. Additionally, several studies have also dealt with the fabrication of hollow zeolite spheres in the absence of sphere templates. Several groups have prepared the hollow zeolite capsules through the multistep assembly of nano-zeolite onto the polymer spheres to form a core-shell structure ${ }^{24-27}$ (or with the further growth in a synthesis ge $^{27}$ ), followed by calcination to remove the template cores. Construction of hollow zeolite with designed structures has attracted considerable attention due to their fast diffusion and low pressure drop while maintaining high catalytic efficiency.

\section{FABRICATION AND CATALYTIC APPLICATION OF ZEOLITE-BASED HOLLOW MATERIALS}

\subsection{Fabrication}

Here, we classify the synthesis methods of zeolite with hollow structures to three categories, including hard template strategy, soft template strategy and template free strategy.

\subsubsection{Hard Template Strategy}

Hard templates are widely used to fabricate inorganic hollow materials. Many compounds, such as polymeric, inorganic nonmetallic, and metallic particles, can be used as hard templates. The final shape and size of the inorganic hollow materials are essentially dependent upon the templates employed. ${ }^{28}$ The hard templates can be categorized to organic template and inorganic template.

2.1.1.1. Polystyrene Template Strategy. Recently, several groups reported on the preparation of shells of zeolite nanocrystals on polystyrene beads using the LBL self-assembly technique. The incompatibility between the organic substrates and the aluminosilicate precursor species is a serious obstacle for the preparation of zeolite layers on such supports by direct hydrothermal synthesis. LBL self-assembly method and hydrothermal synthesis techniques were utilized by Valtchev et al. to prepare hollow silicalite-1. ${ }^{27}$ A schematic presentation of the 


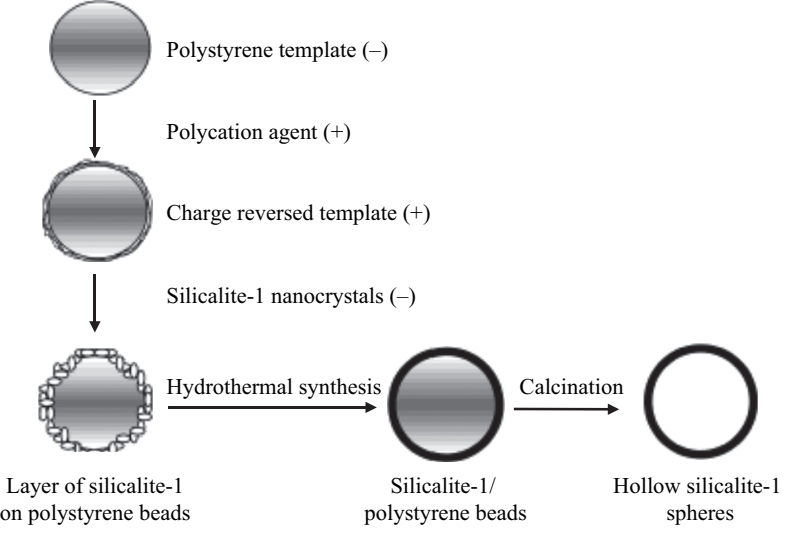

Fig. 1. Schematic representation of the process for the preparation of hollow silicalite-1 spheres. Reprinted with permission from [27], V. Valtchev, Chem. Mater. 14, 4371 (2002). (C) 2002, American Chemical Society.

preparation procedure is shown in Figure 1. The synthesis process of hollow silicalite- 1 sphere is as follows.

(1) The surface charge of the support under investigation was reversed by treatment with the polycation agent;

(2) The charge-reversed beads were then transferred into the colloidal zeolite suspension to electrostatically adsorb nanocrystals;

(3) After the adsorption of silicalite-1 nanocrystals, the beads were subjected to a hydrothermal treatment;

(4) The silicalite-1/polystyrene particles were separated by suction filtration from the colloidal zeolite crystals, washed, and dried. The polystyrene beads and structuredirecting agent were removed by high temperature calcination under oxygen flow.

The corresponding hollow silicalite-1 spheres obtained after calcination were shown in Figure 2. Yang et al. ${ }^{29}$ also used LBL strategy and prepared of hollow zeolite spheres with PS sphere (PSS) as template. First, the positively charged PS sphere templates were prepared by alternately putting as-synthesized PS spheres into the solution of cationic PDDA and anionic PSS to adsorb five (or seven) layer of polyelectrolytes in the order of PDDA/PSS/ PDDA/PSS/PDDA. After every adsorption step, the surplus polyelectrolytes were removed by centrifugation/ water wash/redispersion cycle. Secondly, the nanozeolite and PDDA were alternately adsorbed onto the positively charged PS sphere substrates to form homogeneous nanozeolite/PDDA multilayers. Finally, the hollow zeolite spheres were produced by drying the zeolite coated PS on quartz slides at room temperature, and then calcining them. Wang and coworkers have fabricated hollow spheres of zeolite $\beta$ and silicalite-1 through layer-bylayer self-assembly of nanozeolite-polymer multilayers on PS latex templates, coupled with removal of the core by calcination. ${ }^{25}$ In this way, the zeolite shells are built with well-intergrown crystals so as to improve the mechanical stability and intact ratio of the obtained hollow spheres.

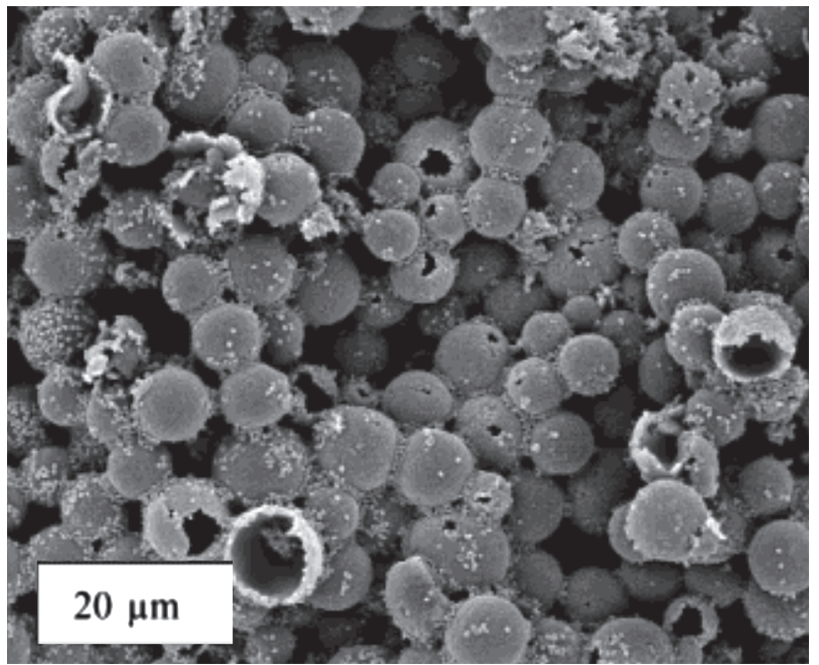

Fig. 2. The corresponding hollow silicalite-1 spheres obtained after calcination (10 $\mu \mathrm{m}$ polystyrene beads as supports). Reprinted with permission from [29], V. Valtchev, Chem. Mater. 14, 4371 (2002). (C) 2002, American Chemical Society.

2.1.1.2. Inorganic Templates. The PS as template to synthesize hollow zeolite based materials had achieved success which can prepare very perfect hollow zeolite structures, nevertheless, this method for the hollow zeolite based materials relied on generated beforehand building block of nanozeolite particles and the LBL technique in which the necessary multistep alternatively coating processes are rather complicated and labor intensive. Several inorganic templates can be used to synthesize hollow zeolite based materials, which include carbon black spheres, $\mathrm{CaCO}_{3}$, fiber, mesoporous silica spheres (MSS), Coal fly ash cenospheres and $\beta$-zeolite crystals.

Chu et al. ${ }^{30}$ had reported the synthesis of hollow hierarchical MCM-22 microspheres (simplified as MCM-22-HS) by a facile one-pot method with carbon black spheres as core-template. A clearer description of the formation process is illustrated in Figure 3. The formation processes of MCM-22-HS were included the process of self-assembly and hydrothermal crystallization.

Wang and coworkers ${ }^{31}$ used cubic or spherical $\mathrm{CaCO}_{3}$ and $\mathrm{Fe}_{3}\left(\mathrm{SO}_{4}\right)_{2}(\mathrm{OH})_{5} \cdot 2 \mathrm{H}_{2} \mathrm{O}$ as templates and the hollow zeolite were achieved by encapsulated zeolite microcapsules, controlled release and conversion of the encapsulated guest species inside the cavity. Three steps are included in the process. First, core particles and silicalite-1 nanocrystals were prepared, and $\mathrm{CaCO}_{3}$ particles with cubic and spherical morphologies were synthesized. $\mathrm{Fe}_{3}\left(\mathrm{SO}_{4}\right)_{2}(\mathrm{OH})_{5} \cdot 2 \mathrm{H}_{2} \mathrm{O}$ particles were produced by hydrothermal treatment of $\mathrm{Fe}_{2}\left(\mathrm{SO}_{4}\right)_{3}$ and urea solution mixtures. Secondly, zeolite microcapsules were fabricated. The core particles were first modified by a three layer polyelectrolyte film (PDDA/PSS/PDDA) which provided core particles with a positively charged outer surface. Then, the negatively charged silicalite- 1 nanocrystals 


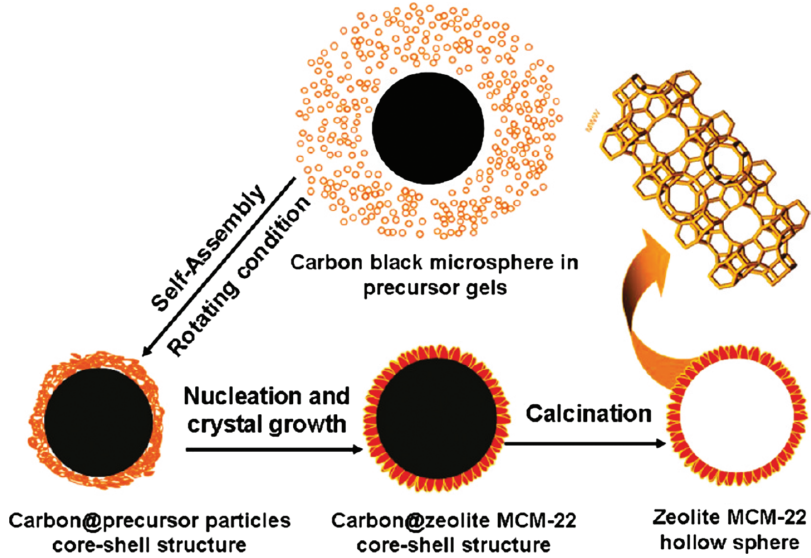

Fig. 3. Schematic representation of the growth process of the MCM-22HS. Reprinted with permission from [30], N. Chu, et al., Chem. Mater 22, 2757 (2010). (C) 2010, American Chemical Society.

were deposited onto the polyelectrolyte modified core particles through electrostatic interactions. Unbound zeolite seeds were removed by washing and centrifugation. Afterwards, the seed-coated core particles were dispersed in a solution containing a template free gel with the composition $28 \mathrm{Na}_{2} \mathrm{O}: 1.5 \mathrm{Al}_{2} \mathrm{O}_{3}: 100 \mathrm{SiO}_{2}: 4000 \mathrm{H}_{2} \mathrm{O}$ (molar ratio) in a Teflon-lined autoclave. The autoclave was closed and heated in an oven at $180{ }^{\circ} \mathrm{C}$ for different periods of time (6-12 h). Finally, hydrochloric acid was used to dissolve the $\mathrm{CaCO}_{3}$ core.

Construction of zeolite coated fibers and hollow zeolite fibers with designed structures has attracted considerable attention due to their fast diffusion and low pressure drop while maintaining high catalytic efficiency. ${ }^{32-35} \mathrm{Ke}$ and coworkers ${ }^{21}$ fabricated hollow zeolite fibers by electrophoretic deposition of nanozeolites onto carbon fibers, then by calcination to remove the substrates of zeolite coated fibers.

Mesoporous silica spheres as hard template were used to prepared hollow zeolite sphere by hydrothermal transformation of the zeolite nano-seeds. ${ }^{22,23,36-40}$ The schematic fabrication strategy was as illustrated in Figure 4. Firstly, the guest species for encapsulation were introduced into the mesopores of pre-synthesized MSS by an impregnation technique followed by calcination or polymerization. Secondly, the guest-incorporated MS spheres were coated with one layer of silicalite-1 nanocrystals via the electrostatic attraction technique. Thirdly, the seeded guest-incorporated MS spheres were treated by a simple hydrothermal transformation process in a tetrapropylammonium hydroxide (TPAOH) aqueous solution for the preparation of discrete guest-encapsulated hollow zeolite spheres or in a TPAOH tetraethoxysilane (TEOS) aqueous solution for the preparation of macroporous monoliths built of interconnected guest-encapsulated hollow zeolite spheres. ${ }^{36}$ Zhang and coworkers ${ }^{41}$ reported a method using the dry-gel conversion approach to fabricate hollow nanospheres with homogeneous and dense shells. They

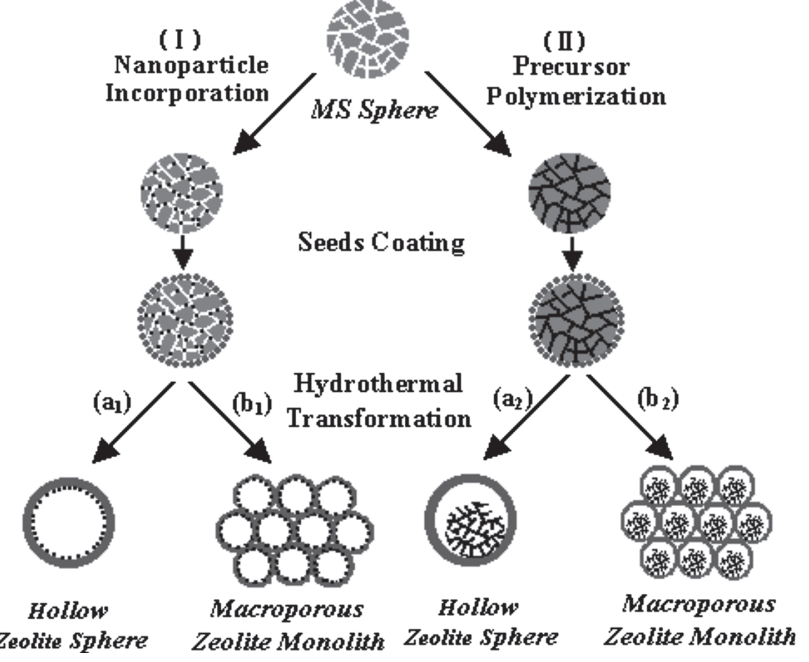

Fig. 4. Schematic illustration for the encapsulation of nano- (I) and micrometer (II) sized particles into discrete hollow zeolite spheres (a1, a2) and macroporous zeolite monoliths (b1, b2). Reprinted with permission from [36], A. G. Dong, et al., Adv. Funct. Mater. 13, 943 (2003). (C) 2003, Wiley-VCH Verlag GmbH.

prepared the hollow spheres by two steps. First, mesoporous solid MCM-41 spheres are synthesized. Second, tetrapropylammonium, that is commonly used to direct the synthesis of MFI-type zeolite, is introduced to transform the MCM-41 spheres into hollow spheres by dry-gel conversion. The MS spheres provide not only a template but also a silica source for capsule shells formation but also a transport medium for guest encapsulation. Hollow spheres composed of nano-sized ZSM-5 crystals via laser ablation were also fabricated using spherical mesoporous DAM-1 or SBA-15 as the silicon source and substrate. ${ }^{42}$ The pulsed laser deposition (PLD) coating is composed of proto-zeolite like fragments which reorganize to form crystalline ZSM-5. The PLD technique offers some advantages over conventional seeding methods such as the convenient PLD coating of 3-D objects and control over the coating thickness.

Hard templating synthesis is an environmentally sound and resource-saving strategy towards the advanced mechanically stable hollow sphere-shaped zeolites, by using hollow glassy aluminosilicate microspheres as the template cores and a nutrition provider. Coal fly ash cenospheres, the by-products of powdered coal combustion, ${ }^{43,44}$ were reported as a suitable starting material for the syntheses of hollow sphere-shaped zeolites. ${ }^{45,46}$ Wang et al. ${ }^{47,48}$ proposed a seed-induced in-situ hydrothermal conversion technique to prepare novel hollow microspheres with zeolite/mullite composite shells from fly ash cenosphere (FAC). Two groups of hollow microspheres were prepared, one with zeolite FAU/mullite composite shells and the other with zeolite LTA/mullite composite shells. Vereshchagina et al. ${ }^{45}$ used one-step procedure to fabricate hollow aluminosilicate microspheres with a composite $\mathrm{NaA}(\mathrm{NaX}) / \mathrm{glass} / \mathrm{mullite}$ composite shell by the direct 
conversion of low-silica cenospheres $(\mathrm{Si} / \mathrm{Al}=1.8)$ separated from coal fly ash. The FAC acts as both the templates and the reactants for the formation of hollow spheres. The zeolite seeds play an important induction role for the conversion of FAC.

Zheng et al. ${ }^{49}$ reported the synthesis of a hollow sphere zeolite composite by using $\beta$-zeolite crystals as silicon sources for the synthesis of Y-zeolite as well as the filler template for the formation of hollow spheres. The mechanism of the formation of the hollow sphere zeolite composite is suggested as follow. Y-zeolite nanoseeds are first adsorbed on the external surface of $\beta$-zeolite crystal due to its higher surface area, which is a very important step for the formation of the hollow spheres. Second, the nanoseeds begin growing because of the interaction between aluminum species from the synthesis solution and silicon species extracted from $\beta$-zeolite crystal, resulting in the formation of Y-zeolite polycrystalline shell around $\beta$-crystal, and $\beta$-zeolite crystal is, therefore, embedded into the shell. Subsequently, the shell layer zeolites become thicker and thicker along with the extraction of silicon from the $\beta$-zeolite crystal. Finally, $\beta$-zeolite crystal can even be exhausted and a cavity whose dimension is close to that of $\beta$-crystal is created in the shell where $\beta$-zeolite particles used to reside. Thus, $\beta$-zeolite crystal not only serves as a source of nutrients for the growth of Y-zeolite crystals but also acts as the filler template for the formation of hollow spheres.

\subsubsection{Soft Template Strategy}

Usually, the hard template strategy can control the spherical morphology and the size of the composites. However, due to its time-/labor-consuming pre-separation process of pure zeolite nanoparticles, this method is limited to the laboratory scale. Soft template-based methods hold appeal because the templates are relatively easy to remove and the synthesis process is relatively simple. However, the morphology and monodispersity of the as-prepared hollow products are usually poor due to the deformability of the soft templates. Controlling monodispersity and spherical morphology of the inorganic hollow structures is the most challenging part of this technique. ${ }^{28}$

Yue and coworkers first synthesized hollow zeolite spheres of silicalite-1 using oil/water emulsions as templates. ${ }^{50}$ The oil-in-water $(\mathrm{O} / \mathrm{W})$ emulsions contain toluene, water and silicalite-1 nanocrystals, in which the toluene was as the template and ensured the zeolitic shells remained intact after hydrothermal treatment, and silicalite-1 nanocrystals act as both the emulsifier and stabilizer. The fabrication procedure is depicted in Figure 5. First, the silicalite-1 stable emulsion was prepared by emulsifying two-phase including the oil and the silicalite-1 seed solution. Then, the lower clear solution was replaced by silicalite-1 synthesis gel as the nutrition. After hydrothermal treatment, the gaps between the zeolite seeds of the bubbles were intergrown in the emulsion, and the crystal growth direction could be from outside to inside. The seed layer kept monolayered and spherical in the process of emulsification, and had been maintained during followed hydrothermal treatment. As a result, the stable hollow spheres of silicalite-1 with monolayered, homogeneous and dense zeolitic shells were prepared based on the emulsion composition for the first time. Interestingly, the oil phase acts as a template in the formation of sphere, so the interiors can be functionalized by dissolving the guest molecules in the oil. The fabrication of hollow zeolite microspheres with ZSM-5 structure and mesoporous shell was reported using the techniques combining sol-gel process in oil-water-oil $(\mathrm{O} / \mathrm{W} / \mathrm{O})$ multiple emulsions and VPT method. ${ }^{51}$ They firstly obtained the amorphous aluminosilicate hollow spheres using the sol-gel technique in O/W/O emulsions. Then they used VPT method to transfer the amorphous aluminosilicate hollow spheres into hollow zeolite microspheres. Wang and coworkers successfully prepared nano-zeolite IM-5 in hydrothermal system with the aid of PEG and CTAB. The formation mechanism of IM-5 may be described as "formation of hydrophobic gel particles and crystallization on its surface." 52

Improved Polymerization-induced colloid aggregation method was developed to prepare zeolite microspheres (ZSMs) with a hierarchical porous structure and a uniform size, which could easily be carried out within half an hour by simply adding urea and formaldehyde into an acidic $\mathrm{pH}$-precontrolled colloidal solution as-obtained from a hydrothermal crystallization process. This procedure successfully avoided the repeated ultrasonic washing and high-speed centrifugation of the nanozeolite colloid during the traditional PICA method, and thereby became energy and time-economical. The structure, morphology and composition of ZSMs can be finely controlled by variation of the acidity in the synthetic system during the polymerization. ${ }^{5}$

\subsubsection{Template-Free Strategy}

Naik and coworkers reported a simple approach for the preparation of hollow spheres with a 10-20 nm thick shell of silicalite-1 nanocrystals. When the nanocrystals of 10-20 nm were dispersed and sonicated in ammoniac ethanol, the nanocrystals were found to self-assemble into hollow spheres. ${ }^{53} \mathrm{~A}$ novel and facile method for the fabrication of zeolite analcime with hierarchical core-shell or hollow icositetrahedro architecture was established by Chen and coworkers. It is demonstrated that the formation of the single crystalline polyhedra does not start from a nucleus and extend outward. It actually follows a reversed route based on oriented aggregation of nanocrystallites, i.e., recrystallization starts at the surface of the polycrystalline microspheres and extends inward. ${ }^{54}$

Alkaline treatment allows synthesizing hollow zeolites on the low or sub-micrometer length scale. Fodor et al. used alkaline treatment and synthesize hollow ZSM-5 nanocrystals with an average size of 100 and $60 \mathrm{~nm}$. 


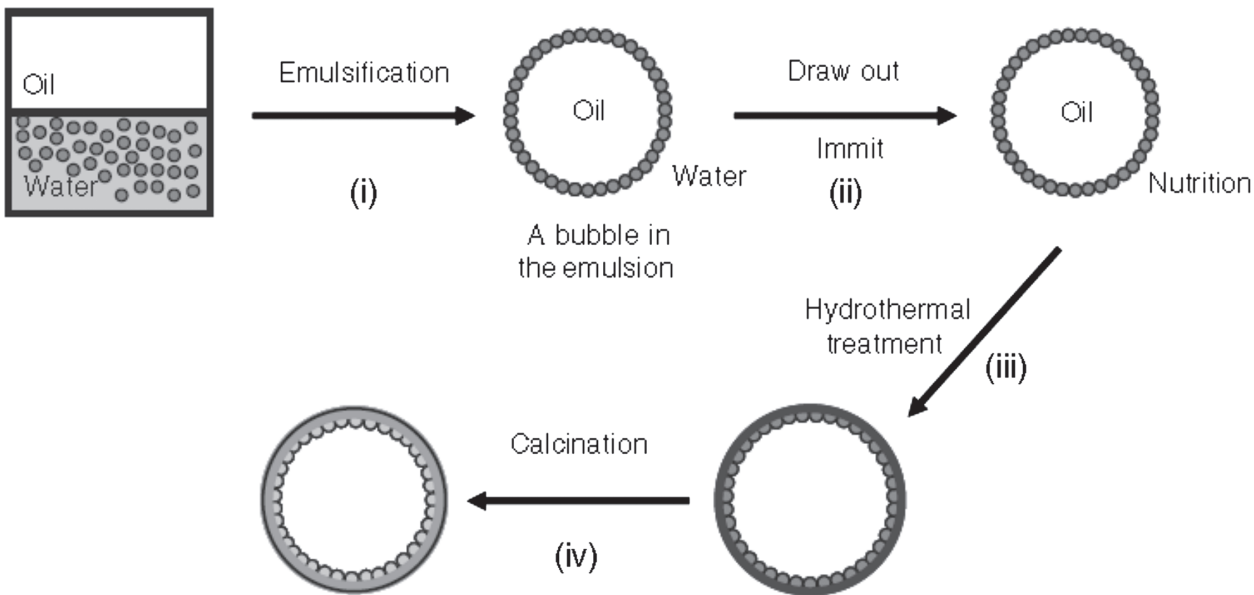

Fig. 5. The fabrication procedure of hollow zeolite spheres. Reprinted with permission from [50], N. Yue, et al., Inorg. Chem. Commun. 14, 1233 (2011). (C) 2011, Elsevier Science BV.

The products have the same size as the respective parent samples, a single hollow in the crystal interior and an approximately $15 \mathrm{~nm}$ single crystal shell as indicated by the presence of uninterrupted zeolite channels. ${ }^{55}$ A kind of hollow mesoporous zeolite microspheres with secondary mesopores on the shell was synthesized by an efficient post steam-assisted crystallization and mild alkaline etching approach (SAC-MAE). Herein, SAC and MAE have been demonstrated as effective routes to synthesize mesoporous zeolite structure (MZS) and hollow zeolite boxes, respectively. ${ }^{56-58}$ The fabrication strategy of MZS involves two key steps: first, the meso-/micropore-structured zeolites with spherical morphology were synthesized under steaming; and subsequently, hollow core was created by selective mild desilication in the inner part of the spheres with aqueous $\mathrm{Na}_{2} \mathrm{CO}_{3}$ solution, which finally leads to hollow mesoporous zeolite microspheres with uniform hollow architecture and unique mesoporous zeolite shells. Groen and coworkers ${ }^{59}$ showed that by controlled desilication of both large and small ZSM-5 crystals they created hollow zeolite architectures. They showed that, when subjected to controlled desilication conditions, $\mathrm{Al}$ gradients in both large and small ZSM-5 crystals induce the formation of hollow particles with a well-preserved Al rich exterior, further confirming the crucial role of $\mathrm{Al}$ in the mechanism of Si extraction.

Dissolution/recrystallization process was also used to fabricate hollow zeolites. ${ }^{60,61}$ Wang and coworkers synthesized hollow TS-1 crystals based on a dissolution/ recrystallization process. The process contains two steps. First, the TS-1 was synthesized. Then the TS-1 was dispersed in a solution containing TPAOH and water. The mixture was then transferred into a Teflon-liner stainless-steel autoclave and heated for certain time under static conditions. The autoclave was then rapidly cooled and the zeolite was recovered by centrifugation, washed with distilled water and dried. It was finally calcined in air. ${ }^{61}$ ZSM-5 nanoboxes with uniform intracrystalline hollow structures were obtained from calcined silicalite-1 nanocystals. ${ }^{62}$ The formation of hollow structures results from a preferential dissolution of the core of the crystals, followed by a re-crystallization in the presence of templating molecules. Generally, a unique hole is obtained, with faces parallel to those of the original crystal. Hollow crystals look like perfectly closed nanoboxes, with a wall thickness in the $20-40 \mathrm{~nm}$ range. During re-crystallization, it is possible to modify the chemical composition of the zeolite without modifying the geometry of the crystal. In particular, starting from silicalite-1, hollow ZSM-5 can be obtained with $\mathrm{Si} / \mathrm{Al}$ ratios as low as 50 .

Hollow aluminosilicate zeolite beta was successfully synthesized by adding CIT-6, that is, zincosilicate zeolite, which has the same topology as beta, as seeds to the $\mathrm{Na}$ aluminosilicate gel without the need for organic structure directing agents. One important factor in the successful organic structure directing agent (OSDA)-free synthesis of hollow beta crystals is the solubility of the seed crystals in alkaline media. ${ }^{63}$

CIT-6 was less stable than aluminosilicate zeolite beta in alkaline media and the solubility changed depending on whether the crystals were calcined or not. Figure 6 shows the TEM image of the sliced crystal. The crystal growth proceeded from the outside inward after the complete dissolution of the CIT- 6 seed crystal. ${ }^{63}$

\subsection{Catalytic Applications}

The most attractive characteristics of zeolite based hollow materials are their well-defined morphology, their large specific surface area and low density. Hollow zeolite based materials are used as catalysts in many catalytic reactions, such as catalytic cracking, ${ }^{64}$ hydroxylation, ${ }^{61}$ SuzukiMiyaura cross-coupling reaction, ${ }^{37}$ aldol condensations, ${ }^{65}$ dehydration, ${ }^{5}$ methane dehydroaromatization, ${ }^{30}$ hydrodesulfurization, ${ }^{41}$ Baeyer-Villiger oxidation ${ }^{61}$ and so on.

Hollow titanium silicalite (HTS) zeolite has been employed to catalyze the Baeyer-Villiger oxidation 


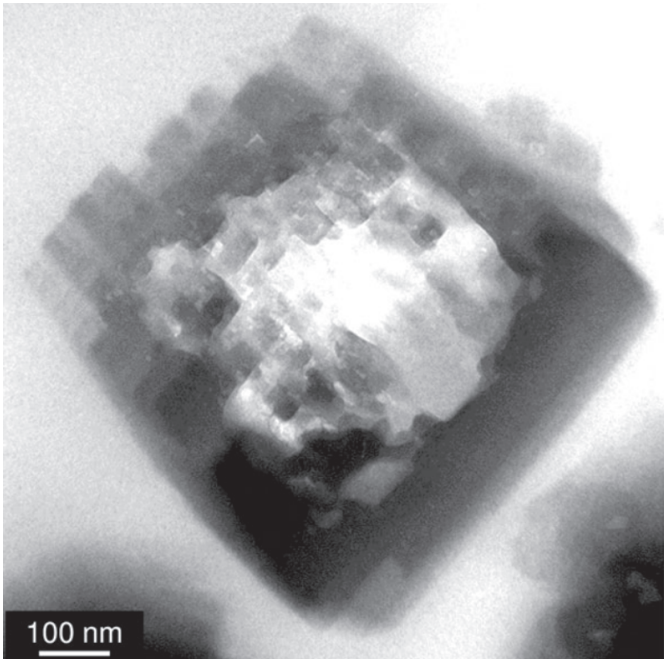

Fig. 6. TEM image of the sliced crystal. Reprinted with permission from [36], K. Iyoki, et al., Chem. Asian J. 8, 1419 (2013). (C) 2013, Wiley-VCH Verlag GmbH.

of cyclohexanone. Both cyclohexanone conversion and desired product distribution obtained in the presence of HTS zeolite are different from those obtained on TS-1 zeolite as reported, which might be ascribed to the difference in their mesopore volume. ${ }^{66}$ The cyclohexanone oxidation reaction catalyzed by HTS zeolite is a representative consecutive reaction, the main target products of which are $\varepsilon$-caprolactone, 6-hydroxyhexanoic acid and adipic acid. Xia and coworkers have studied the effect of $\mathrm{H}_{2} \mathrm{O}_{2}$ /cyclohexanone mole ratio on the cyclohexanone conversion, the total target product selectivity, the distribution of selectivity of three target products and their variations with reaction time which indicate that HTS zeolite shows a high performance for the BaeyerVilliger reaction of cyclohexanone and catalytic oxidation of 6-hydroxyhexanoic acid under mild condition. ${ }^{60}$ The hollow zeolite encapsulated with noble metal nanoparticles were used as catalyst in a series of catalytic oxidation reactions of alcohols. Compared to commercial catalysts, such as $\mathrm{Pt} / \mathrm{SiO}_{2}$ and $\mathrm{Pt} / \mathrm{Al}_{2} \mathrm{O}_{3}$, the zeolite based hollow catalyst retained most of their reactivity even in the presence of a significant amount of poison contaminants. ${ }^{38}$ The hollow zeolitic sphere was examined as supports for $\mathrm{Ni}$ and $\mathrm{Mo}$ oxides as catalyst in hydrodesulfurization of FCC gasoline. The sulfur removal was promoted while the olefin hydrogenation of FCC gasoline was suppressed and thus the octane number was preserved when the hollow spheres (Na type) were used as support compared with conventional ZSM-5. ${ }^{41}$

Hollow zeolite microspheres were used as catalyst in the reaction of alkylation of toluene with benzyl chloride, it was found that hollow zeolite microspheres were highly active compared to conversional ZSM-5. The highest toluene conversion (49.8\%) is achieved over hollow zeolite microspheres, while the conversional ZSM-5 (24.8\%) displays the lowest conversion. ${ }^{67}$

Methane dehydroaromatization (MDA) reaction which was considered as a promising route for direct conversion of methane into high value-added chemicals has attracted increasing attentions in the past several decades. Chu and coworkers synthesized nest-like hollow hierarchical MCM-22 microspheres and the catalytic properties of the catalyst in the reaction of MDA were tested. ${ }^{30} \mathrm{MCM}$ 22-HS exhibited significantly enhanced benzene yield and catalyst lifetime in MDA reaction. It is believed that the exceptional catalyst performance is due to hierarchical and hollow structure. The higher external surface and hollow structure resulting from mesorpores offered an important effect on improving the high benzene yield and catalyst lifetime by generating more catalysis active sites by means of promoting catalyst transport onto the reaction sites and by improving diffusion of larger product through the zeolite catalyst, respectively. The sites associated with catalyst deactivation could also be reduced, which was contributed to improvement of the catalyst lifetime.

\section{CONCLUSION}

In this article, we summarized the fabrication methods and catalytic application for zeolite based hollow materials. The mechanisms for synthesizing zeolite based hollow materials were depicted in this review. For hard template strategy, the templates of PS spheres and MSS were in common use. The emulsion method was used in the soft template strategy, and the interiors of such hollow spheres can be functionalized by changing the soluble guests in the oil which can be used to synthesize multi-functional interiors spheres. Self-assembly, alkaline treated method and dissolution/recrystallization process were the mainly used methods. Through the alkaline treated process we can get zeolite based hollow materials with low or sub-micrometer length scale. The catalytic applications of these zeolite based hollow materials have a broad prospect due to the advantage of the zeolite based hollow materials.

Acknowledgment: The project was sponsored by "100 Talents" program of Chinese Academy of Sciences (Grant KJCX2-EW-H05) and Doctoral Fund of Shandong Province (BS2013CL029).

\section{References and Notes}

1. M. Stocker, Micropor. Mesopor. Mater. 82, 257 (2005)

2. A. Hagen and F. Roessner, Catal. Rev. Sci. Eng. 42, 403 (2000).

3. W. W. Kaeding, L. B.Young, and C. C. Chu, J. Catal. 89, 267 (1984).

4. F. G. Dwyer and T. F. Degnan, Stud. Surf. Sci. 76, 499 (1993).

5. Y. Shi, X. Li, J. Hu, J. Lu, Y. Ma, Y. Zhang, and Y. Tang, J. Mater. Chem. 21, 16223 (2011).

6. T. Bein, chem. Mater. 8,1636 (1996).

7. A. Tavolaro and E. Drioli, Adv. Mater. 11, 975 (1999).

8. Y. J. Wang, Y. Tang, A. G. Dong, X. D. Wang, N. Ren, W. Shan, and Z. Gao, Adv. Mater. 14, 994 (2002). 
9. L. Tosheva, B. Mihailova, V. Valtchev, and J. Sterte, Micropor. Mesopor. Mater. 48, 31 (2001).

10. Y. J. Wang, Y. Tang, Z. Ni, W. M. Hua, W. L. Yang, X. D. Wang, W. C. Tao, and Z. Gao, Chem. Lett. 29, 510 (2000).

11. H. T. Wang, L. M. Huang, Z. B. Wang, A. Mitra, and Y. S. Yan, Chem. Commun. 37, 1364 (2001).

12. Y. J. Lee, J. S. Lee, Y. S. Park, and K. B. Yoon, Adv. Mater. 13, 1259 (2001).

13. A. A. Dong, Y. J. Wang, Y. Tang, Y. H. Zhang, N. Ren, and Z. Gao, Adv. Mater. 14, 1506 (2002).

14. H. C. Xin, X. P. Li, L. Chen, Y. Huang, G. R. Zhu, and X. B. Li, Energy Environ. Focus 2, 18 (2013).

15. M. D. Shultz, M. J. Bowman, Y. W. Ham, X. M. Zhao, G. Tora, and J. Chmielewski, Angew. Chem. Inter. Ed. 39, 2710 (2000).

16. S. Komarneni, H. Katsuki, and S. Furuta, J. Mater. Chem. 8, 2327 (1998).

17. H. Xin, A. Koekkoek, Q. Yang, R. van Santen, C. Li, and E. J. M Hensen, Chem. Commun. 45, 7590 (2009).

18. H. Xin, J. Zhao, S. Xu, J. Li, W. Zhang, X. Guo, E. J. M. Hensen, Q. Yang, and C. Li, J. Phys. Chem. C 114, 6553 (2010).

19. H. C. Xin, X. P. Li, Y. Fang, X. F. Yi, W. H. Ju, Y. Y. Chu, F. Zhang, A. M. Zheng, H. P. Zhang, and X. B. Li, J. Catal. 312, 204 (2014).

20. Y. J. Wang, Y. Tang, X. D. Wang, W. L. Yang, and Z. Gao, Chem Lett. 29, 1344 (2000)

21. C. Ke, W. L. Yang, Z. Ni, Y. J. Wang, Y. Tang, Y. Gu, and Z. Gao, Chem. Commun. 37, 783 (2001).

22. A. G. Dong, Y. J. Wang, Y. Tang, N. Ren, Y. H. Zhang, and Z. Gao, chem. Mater. 14, 3217 (2002).

23. Z. Wang, Y. Liu, J. G. Jiang, M. He, and P. Wu, J. Mater. Chem 20, 10193 (2010).

24. K. H. Rhodes, S. A. Davis, F. Caruso, B. J. Zhang, and S. Mann Chem. Mater. 12, 2832 (2000)

25. X. D. Wang, W. L. Yang, Y. Tang, Y. J. Wang, S. K. Fu, and Z. Gao, Chem. Commun. 36, 2161 (2000).

26. V. Valtchev and S. Mintova, Micropor. Mesopor. Mater. 43, 41 (2001).

27. V. Valtchev, Chem. Mater. 14, 4371 (2002).

28. J. Hu, M. Chen, X. Fang, and L. Wu, Chem. Soc. Rev. 40, 5472 (2011).

29. W. L. Yang, X. D. Wang, Y. Tang, Y. J. Wang, C. Ke, and S. K. Fu, J. Macromol. Sci. Pure Appl. Chem. 39, 509 (2002).

30. N. Chu, J. Wang, Y. Zhang, J. Yang, J. Lu, and D. Yin, chem. Mater. 22, 2757 (2010)

31. D. J. Wang, G. B. Zhu, Y. H. Zhang, W. L. Yang, B. Y. Wu, Y. Tang, and Z. K. Xie, New J. Chem. 29, 272 (2005).

32. Z. Hong, F. Sun, D. Chen, C. Zhang, X. Gu, and N. Xu, Int. J. Hydro. Energy 38, 8409 (2013).

33. L. Lai, J. Shao, Q. Ge, Z. Wang, and Y. Yan, J. Membr. Sci. 409, 318 (2012).

34. Z. Deng, C. H. Nicolas, Y. Guo, A. Giroir-Fendler, and M. PeraTitus, Sep. Purif. Technol. 80, 323 (2011).

35. L. Shan, J. Shao, Z. Wang, and Y. Yan, J. Membr. Sci. 378, 319 (2011).

36. A. G. Dong, N. Ren, W. L. Yang, Y. J. Wang, Y. H. Zhang, D. J. Wang, H. H. Hu, Z. Gao, and Y. Tang, Adv. Funct. Mater. 13, 943 (2003).

37. Z. Guan, J. Hu, Y. Gu, H. Zhang, G. Li, and T. Li, Green Chem 14, 1964 (2012)
38. N. Ren, Y. H. Yang, J. Shen, Y. H. Zhang, H. L. Xu, Z. Gao, and Y. Tang, J. Catal. 251, 182 (2007).

39. N. Navascues, C. Tellez, and J. Coronas, Micropor. Mesopor. Mater. 112, 561 (2008).

40. C. Casado, J. Bosque, N. Navascues, C. Tellez, and J. Coronas, Micropor. Mesopor. Mater. 120, 69 (2009).

41. J. Zhang, G. Wang, F. Jin, X. Fang, C. Song, and X. Guo, J. Colloid Inter. Sci. 396, 112 (2013).

42. C. R. Xiong, D. Coutinho, and K. J. Balkus, Micropor. Mesopor. Mater. 86, 14 (2005).

43. V. B. Fenelonov, M. S. Mel'gunov, and V. N. Parmon, Kona Powder Part. J. 189 (2010).

44. N. N. Anshits, O. A. Mikhailova, A. N. Salanov, and A. G. Anshits, Fuel 89, 1849 (2010).

45. H. Tao, J. Yao, L. Zhang, and N. Xu, Mater. Lett. 63, 203 (2009).

46. T. A. Vereshchagina, N. N. Anshits, O. M. Sharonova, N. G. Vasil'eva, S. N. Vereshchagin, N. N. Shishkina, E. V. Fomenko, and A. G. Anshits, Glass Phys. Chem. 34, 547 (2008).

47. D. J. Wang, Y. Tang, A. G. Dong, Y. H. Zhang, Y. J. Wang, Chin. Chem. Lett. 14, 1299 (2003).

48. T. A. Vereshchagina, S. N. Vereshchagin, N. N. Shishkina, O. A. Mikhaylova, L. A. Solovyov, and A. G. Anshits, Micropor. Mesopor. Mater. 169, 207 (2013).

49. J. Zheng, Q. Zeng, J. Ma, X. Zhang, W. Sun, and R. Li, Chem. Lett. 39, 330 (2010).

50. N. Yue, M. Xue, and S. Qiu, Inorg. Chem. Commun. 14, 1233 (2011).

51. J. Cheng, S. Pei, B. Yue, L. Qian, C. He, Y. Zhou, and H. He, Micropor. Mesopor. Mater. 115, 383 (2008).

52. L. Wang, W. Yang, F. Ling, Z. Shen, R. Yang, W. Sun, X. Fang, and H. Ji, Micropor. Mesopor. Mater. 163, 243 (2012).

53. S. P. Naik, A. S. T. Chiang, R. W. Thompson, and F. C. Huang, chem. Mater. 15, 787 (2003).

54. X. Chen, M. Qiao, S. Xie, K. Fan, W. Zhou, and H. He, J. Am. Chem. Soc. 129, 13305 (2007).

55. D. Fodor, L. Pacosova, F. Krumeich, and J. A. van Bokhoven, Chem. Commun. 50, 76 (2014).

56. J. Zhou, Z. Hua, W. Wu, Z. Liu, Y. Zhu, Y. Chen, and J. Shi, dalton T 40, 12667 (2011)

57. C. Mei, P. Wen, Z. Liu, H. Liu, Y. Wang, W. Yang, Z. Xie, W. Hua, and Z. Gao, J. Catal. 258, 243 (2008).

58. C. Mei, Z. Liu, P. Wen, Z. Xie, W. Hua, and Z. Gao, J. Mater. Chem. 18, 3496 (2008).

59. J. C. Groen, T. Bach, U. Ziese, A. Donk, K. P. de Jong, J. A. Moulijn, and J. Perez-Ramirez, J. Am. Chem. Soc. 127, 10792 (2005).

60. C. Xia, B. Zhu, M. Lin, and X. Shu, China Pet. Process Pe. 14, 33 (2012).

61. Y. Wang, M. Lin, and A. Tuel, Micropor. Mesopor. Mater. 102, 80 (2007).

62. Y. Wang and A. Tuel, Micropor. Mesopor. Mater. 113, 286 (2008).

63. K. Iyoki, K. Itabashi, and T. Okubo, Chem. Asian J. 8, 1419 (2013).

64. J. Li, D. Zhang, Q. Gao, Y. Xu, D. Wu, Y. Sun, J. Xu, and F. Deng, Mater. Chem. Phys. 125, 286 (2011).

65. J. Zhao, Z. Hua, Z. Liu, Y. Li, L. Guo, W. Bu, X. Cui, M. Ruan, H. Chen, and J. Shi, Chem. Commun. 45, 7578 (2009).

66. A. Bhaumik, P. Kumar, and R. Kumar, Catal. Lett. 40, 47 (1996).

67. H. Tao, J. Ren, X. Liu, Y. Wang, and G. Lu, J. Solid State Chem. 200, 179 (2013). 